\title{
A Review on the Preparation Method of Fruit Leathers
}

\author{
M. Sai Srinivas ${ }^{1 *}$, S. K. Jain ${ }^{1}$, N. K. Jain ${ }^{1}$, S. S. Lakhawat ${ }^{2}$, \\ Arun Kumar ${ }^{3}$ and H. K. Jain ${ }^{2}$ \\ ${ }^{1}$ College of Technology and Engineering, MPUAT, Udaipur-313001, Rajasthan, India \\ ${ }^{2}$ Rajasthan College of Agriculture, MPUAT, Udaipur-313001, Rajasthan, India \\ ${ }^{3}$ College of Dairy and Food Technology, MPUAT, Udaipur-313001, Rajasthan, India \\ *Corresponding author
}

\begin{abstract}
A B S T R A C T
Fruit leathers are made by drying a very thin layer of fruit puree to produce a product with a texture similar to soft leather. Fruit leather is the product prepared by blending fruit purees or pulp extracted from ripe pulpy fruit, sugar or other nutritive sweeteners and other ingredients and additives desired for product and dehydrated to form sheet which can be cut to desired shape and size. The preservation of fruit leathers depends on their low moisture content, the natural acidity of the fruit used and high sugar contents. Various drying systems including combined convective and far-infrared drying, hot air drying, microwave drying, solar drying, and sun drying have been used to make fruit leathers. Most fruit leathers are dried at 30 to $80^{\circ} \mathrm{C}$ for up to 24 hours until the target final moisture content $(12-20 \%)$ has been reached. Research about fruit leathers began in the 1970s. This paper reviews the literature on fruit leathers; most notably it covers various facets of process and development of fruit leathers.
\end{abstract}

Keywords

Fruit leather,

Drying, Blending,

Moisture content

Article Info

Accepted:

05 April 2020

Available Online:

10 May 2020

\section{Introduction}

Fruit leathers are dried sheets of fruit pulp which have a soft, rubbery texture and a sweet taste (Diamante et al., 2014). Leathers can also be made from a mixture of fruits and also eaten as snack foods instead of boiled sweets. It is chewy and flavourful, naturally low in fat and high in fiber and carbohydrates; it is also lightweight and easily stored and packed
(Ayotte, 1980; Diamante et al., 2014). Consuming fruit leather is an economic and convenient value-added substitute for natural fruits as a source of various nutritional elements. They are produced by dehydrating of fruit puree into a leathery sheet (Raab and Oehler, 1999). The edible portion of fruit (one or more types) is pureed, mixed with other ingredients to improve its physicochemical and sensory characteristics, heated, formed 
(flattened and shaped) and then dried on a flat trays until a cohesive fruit leather is obtained. Fruit pulp-based fruit leathers are nutritious and organoleptically acceptable to customers. They contain substantial quantities of dietary fibers, carbohydrates, minerals, vitamins, and antioxidants (which remain constituents of the finished product) (Ayotte, 1980; Gujral and Brar, 2003; Diamante et al., 2014). Most fresh fruits have a short harvest season and are sensitive to deterioration and even when stored under refrigerated conditions; therefore, making fruit leather from fresh fruits is an effective way to preserve fruits (Maskan et al., 2002; Diamante et al., 2014). Fruit leathers are easy to prepare and a good way to use left-over canned fruit and slightly over-ripe fresh fruit. Most fruit or combinations of fruits can be used. Apricots, apples, grapes, berries, bananas, pineapples, oranges, pears, peaches, plums, melons, and most tropical fruits can be blended and dried to make fruit leathers.

In addition, mixed fruit leathers like guava and papaya fruit leather are also available. Basically, fruit pulps are mixed with appropriate quantities of sugar, pectin, acid, and colour and then dried into sheet-shaped products (Diamante et al., 2014). Gujral and Brar, (2003) added sugars and pectin to mango leathers. The sugar gave the product a sweeter taste and increased the solids content; then pectin was used to thicken the pulp, modify the flexible texture, and ensure the retention of the shapes of the dried product. Furthermore, they also prepared mango leather with the addition of potassium meta bisulphite to get better sensory qualities and the results were satisfactory for customers/consumers. Chan and Cavaletto (1978) made papaya leathers with sucrose and sodium bisulphite $\left(\mathrm{SO}_{2}\right)$. They found that $\mathrm{SO}_{2}$ reduced the change in color of the papaya leathers during processing and storage (Diamante et al., 2014).
Various additives can be used, such as glucose syrup, sodium meta bisulphite, and sorbic acid, depending on the types of fruit leather (Demarchi et al., 2013; Quintero Ruiz et al., 2012; Sharma et al., 2013).

Fruit leathers can be dried using various drying method including sun, oven, cabinet or dehydrator drying method ( Raab \& Oehler, 1999). The composition of the final fruit product may vary depending on the processing conditions. Sun-dried products can become discoloured and the process can be unhygienic and lengthy. Hot air drying is an alternative method that needs less drying time and improves the quality of the dried fruit (Maskan et al., 2002). Modern dryers, such as tunnel dryers and forced air circulation cabinet dryers have been used for making fruit leathers with a better colour and flavour. Over $85 \%$ of industrial dryers are of the convective type with hot air or direct combustion gases used as the drying medium. Drying occurs from vaporization of the liquid by supplying heat to the wet material.

Conduction, like contact or indirect dryers, convection such as direct dryers, and radiation or volumetrically by placing the wet material in a microwave or radio frequency electromagnetic fields are various methods that are used in making fruit leathers. The methods chosen are dependent on what kind of fruit and the commercial conditions.

The product changes during drying include shrinkage, puffing, and crystallization. Sometimes there are also desirable or undesirable chemical or biochemical reactions occurring that will cause changes in color, texture, odour, and other properties in the final product. In many processes, incorrect drying methods result in irreversible damage to the quality of the final product which makes the product no saleable (Huang and Hsieh, 2005; Gujral and Brar, 2003). 
With modern dehydrators and well-designed drying methods, fruit leathers can be dried at any time of the year to reach the requirements of customers. In the current growing market of fruit leathers, commercial packaging is necessary. Packaging materials for fruit leather are required to prolong the shelf-life of the product and, normally, relate to the stability of water activity, microbiological stability, sensory properties, and physicochemical characteristics (Irwandi et al., 1998).

\section{Production of fruit leathers}

As the pulping process can be mechanised, it makes this process less labour-intensive. Further it offers a scope for full utilisation of pulp and added ingredients resulting in negligible waste generation and processing losses in comparison to other methods of processing (Tiwari, 2019). The following basic steps are involved at all levels of production of fruit leathers at three scales; from a very small simple home based system, through cottage industry to small industrial production.

Preparation of the puree

Batch preparation

Drying

Packing and storage

\section{Development of fruit leather}

Ripe fruits are used in its preparation. Wash ripe fruits and collect fruit pulp by removing its peel or shell. Addition of water to fruit pulp (if required), mix well and heat it up to $80^{\circ} \mathrm{C}$. Collect fruit pulp free of seeds and fibers by straining heated mass through stainless steel sieve. Add sugar, citric acid and preservatives etc to treated pulp. Boil treated pulp and spread on aluminium trays smeared with butter. Dry at $55-60^{\circ} \mathrm{C}$ for $15-16 \mathrm{hrs}$ to a moisture content of $14.5 \%$. Cut slices of dried pulp in aluminium trays, wrap in butter paper and pack in polyethylene bags. Addition of up to 10 per cent sugar to the extracted pulp was found to be ideal before drying the pulp to a moisture content of 14.5 per cent (Roy and Singh, 1979; Singh and Chaurasiya, 2014).

Mango pulp is commonly used for making fruit leather as mango fruits are usually available in market. It is also known as Ampapar (Hindi) or Tandra (Telugu) (Tiwari, 2019). However, other fruit pulp leather can also be made from guava, papaya, apricot, banana, etc. Different fruits like apple, berries, kiwifruit, and a range of tropical fruit pulp have already been used for making fruit leathers and these are consumed as popular snack (Huang and Hsieh, 2005; Tiwari, 2019). Fruit leathers are usually promoted as natural snacks in health food market. Even dehydrated fruit pulp can be alternatively rehydrated in water after cutting into small pieces to prepare RTS juice by blending (Nanjundaswamy et al., 1976). Dehydrated fruit-based snacks such as fruit leathers have the advantage of being perceived by consumers as healthier food. Therefore, they can be a potential carrier for prebiotics and probiotics. An ideal fruit leather can be prepared from fruits such as apples, pears, pineapples, and strawberries, apricots, cherries, nectarines, berries and peaches etc (Tiwari, 2019). Product similar to fruit leather is also known as pestil in some part of world. Y1lmaz et al., (2017) stated that pestil is a traditional fruit product commonly produced in Anatolia, Arabia, Armenia, Lebanon, Persia and Syria. Pestil is a sweet product with high nutrient content such as mineral, vitamins and considered as a rich energy source because of its high carbohydrate content. It is also known with different names such as 'Bastegh', 'Qamar eldeen', 'Bestil', and 'Fruit Leather'. Pestil is made from different fruits such as apricot, kiwi-fruit, grape, mulberry and pear (Tiwari, 2019). 
Development of fruit leathers by blending technique

Generally reports on fruit leathers are based on use of single type of fruit or pulp. Beside usual process of fruit leather preparation, blending of different fruit pulp for preparation of fruit leathers has also been reported. The main purpose of choosing two or more fruits for blending is improvement in nutritional qualities, sensory qualities (colour, texture and flavour) and storage stability as per the contribution from different fruits during product formulation (Tiwari, 2000; Tiwari, 2019). Further, it also gives an opportunity to utilize fruit which are available throughout the year. Good blended fruit leather from mango-guava pulp has been reported by Hemakar et al., (2000). Cherian and Cheriyan, (2003) carried out works on acceptability study on blended papaya leather.

Flow-chart.1 General process preparation of fruit leather

Fruits (mature, ripe)

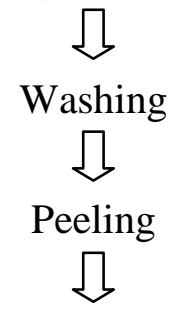

Seed removal and separation into segments<smiles>[CH]1CC1</smiles>

Extraction of fruit pulp<smiles></smiles>

Addition of water to fruit pulp (If required)

$\prod$

Heated up to $80^{\circ} \mathrm{C}$

ఏ

Strained the fruit pulp with stainless steel sieve<smiles>C1=CC=C1</smiles>

Addition of sugar, citric acid and Preservatives etc (If needed)<smiles></smiles>

Boiling of pulp

凸

Spread on aluminium trays smeared with butter

ఏ

Dry at $55-60^{0} \mathrm{C}$ for $15-16 \mathrm{hrs}$ to a moisture content of $14.5 \%$

ڤ

Wrap dried pulp in butter paper and pack in polyethylene bags

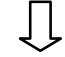

Storage 
Other works relating to acceptable blended fruit bar have been reported by Sreemathi (2008) in sapota-papaya leather; Kaushal and Bhat (1999) in blended fruit leather using sprouted soya slurry with peach, plum and apricot and Chauhan, et al., (1993) in apricotsoy fruit leather. Narayana et al., (2007) reported improvement in the moisture content in banana fruit leather after addition of pectin. Blended fruit leather made from $60 \%$ papaya pulp with $40 \%$ guava pulp was best with improved nutritional and sensory attributes besides being very cost effective (Kumar et al., 2010). Similarly, purees of apple, banana and pineapple have also been used for making mixed fruit leathers (Tiwari, 2019).

In conclusion, fruit leathers, sometimes referred to as fruit rolls or Fruit Roll-ups, are popular dried food snacks. The popularity of the fruit leather has increased significantly in the last 10 years because many view these snacks are more healthful than other confections. Fruit leather is massmanufactured by different companies but can also be made rather easily at home. Making fruit leathers from commercial fruits for example grapes, raspberries, apples, and strawberries, mango, papaya etc on the kitchen stove and using the oven or a food dehydrator to assist in the drying process. In India mainly mango leather is commercially available in the market. Underutilized fruits like bael, ber, jamun etc. can also be used for preparation of fruit leathers and these fruit leathers can be popularised commercially, so that postharvest losses of fruits can be reduced. Fruit leathers can be a good, convenient and natural alternative to junk foods or foods which are with high salt, sugar and fat

\section{References}

Ayotte, E. 1980. Fruit leather. Publication no. P-228, University of Alaska
Cooperative Extension Service,

Fairbanks, Alaska, USA.

Chan, H. T. and. Cavaletto, C. G. 1978. Dehydration and storage stability of papaya leather. Journal of Food Science, 43:1723-1725.

Chauhan, S.K., Joshi, V. K. and Lal, B. B. 1993. Apricot soy fruit bar: a new protein enriched product. Journal of Food Science and Technology, 30 (6): 457-458.

Cherian, B.and Cheriyan, S. 2003. Acceptability study on blended papaya leather. Journal of Food Science and Technology. 40(3):293-295.

Demarchi, S. M.,. Quintero Ruiz, N. A, Concellon, A. and Giner, S. A. 2013. Effect of temperature on hot-air drying rate and on retention of antioxidant capacity in apple leathers. Food and Bio products Processing, 91: 310-318.

Diamante, L.M., Bai, X and Busch, J. 2014. Fruit leathers: method of preparation and effect of different conditions on qualities. International Journal of Food Science 12:1-9.

Gujral, H. S and Brar, S.S. 2003. Effect of hydrocolloids on the dehydration kinetics, color, and texture of mango leather. International Journal of Food Properties, 6(2): 269-279.

Hemakar, A.K., Toma, M.C and Singh, U.B. 2000. Studies on blending of guava pulp with mango pulp for dehydration (Mango-Guava sheet). Indian Food Pack, 45 (4): 45-50.

Huang, X and Hsieh, F.H. 2005. Physical properties, sensory attributes, and consumer preference of pear fruit leather. Journal of Food Science, 70(3):177-186.

Irwandi, J., Che Man, Y. B., Yusof, S., Jinap, S. and Sugisawa, H. 1998. Effects of type of packaging materials on physicochemical, microbiological and sensory characteristics of durian fruit 
leather during storage. Journal of the Science of Food and Agriculture, 76(3): 427-434.

Koushal, B.B.L and Bhat, A. 1999. Studies on physicochemical propertied of fruit leather blended soya slurry. Indian Food Packer, 10:18-23.

Kumar, R., Patil, R.T. and Mondal, G. 2010. Development and evaluation of blended papaya leather, Acta Hortic., 851, 565570.

Maskan, A., Kaya, S. and Maskan, M. 2002. Hot air and sun drying of grape leather (pestil). Journal of Food Engineering, 54( 1): 81-88.

Nanjundaswamy, A. M., Shetty, G. R., Saroja, S. 1976. Studies on the development of newer products from mango. Ind Food Packer, 30: 95-102.

Narayana, C.K., Mustaffa, M.M. and Sathiamoorty, S. 2007. Standardisation of process for preparation of banana fruit bar. Indian Food Pack, 64 (3), 349350.

Quintero Ruiz, N. A., Demarchi, S. M., Massolo, J. F.,. Rodoni, L. M and Giner, S.A. 2012. Evaluation of quality during storage of apple leather. $L W T-$ Food Science and Technology, 47(2): 485-492.

Raab, C and Oehler N. 1999. Making dried fruit leather. Fact Sheet 232, pp 1-4, Oregon State University Extension
Service.

Sharma, S. K., Chaudhary, S. P., Rao, V. K., Yadav, V. K. and Bisht, T. S. 2013. Standardization of technology for preparation and storage of wild apricot fruit bar. Journal of Food Science and Technology, 50: 784-790.

Singh, A. K and Chaurasiya, A. K. 2014. Post-harvest management and value addition in bael (Aegle marmelos Corr.) International Journal of Interdisciplinary and Multidisciplinary Studies 1 (9): 65-77.

Sreemathi, M., Sankaranarayanan, R. And Balasubramanyan, S. 2008. Sapotapapaya bar. Madras Agricultural Journal, 95 (1/6), 170-173.

Tiwari, R B. 2019. Advances in technology for production of fruit bar: A review. Pantnagar Journal of Research, 17(1): 11-18.

Tiwari, R.B. 2000. Studies of blending of guava and papaya pulp for RTS beverage. Indian Food Packer, 54: 6872.

Yılmaz, F.M., Yu- ksekkaya, S., Vardin, H. and Mehmet Karaasla, M. 2017. The effects of drying conditions on moisture transfer and quality of pomegranate fruit leather (pestil). Journal of the Saudi Society of Agricultural Sciences, 16: 33-40.

\section{How to cite this article:}

Sai Srinivas. M., S. K. Jain, N. K. Jain, S. S. Lakhawat, Arun Kumar and Jain. H. K. 2020. A Review on the Preparation Method of Fruit Leathers. Int.J.Curr.Microbiol.App.Sci. 9(05): 773778. doi: https://doi.org/10.20546/ijcmas.2020.905.085 